TITLE:

\title{
Effects of laser polarization on photoelectron angular distribution through laser-induced continuum structure
}

\section{$\operatorname{AUTHOR}(\mathrm{S}):$}

Buica, G; Nakajima, T

\section{CITATION:}

Buica, G ... [et al]. Effects of laser polarization on photoelectron angular distribution through laser-induced continuum structure. PHYSICAL REVIEW A 2005, 72(5): 053416.

\section{ISSUE DATE:}

2005-11

URL:

http://hdl.handle.net/2433/50419

RIGHT:

Copyright 2005 American Physical Society 


\title{
Effects of laser polarization on photoelectron angular distribution through laser-induced continuum structure
}

\author{
Gabriela Buica $^{1, *}$ and Takashi Nakajima ${ }^{1,2, \dagger}$ \\ ${ }^{1}$ Institute of Advanced Energy, Kyoto University, Gokasho, Uji, Kyoto 611-0011, Japan \\ ${ }^{2}$ Institute for Solid State Physics, The University of Tokyo, 5-1-5 Kashiwanoha, Kashiwa, Chiba 277-8581, Japan
}

(Received 12 August 2005; published 30 November 2005)

\begin{abstract}
We theoretically investigate the effects of laser polarization on the photoelectron angular distribution through laser-induced continuum structure. We focus on a polarization geometry where the probe and dressing lasers are both linearly polarized and change the relative polarization angle between them. We find that the total ionization yield and the branching ratio into different ionization channels change as a function of the relative polarization angle, and accordingly the photoelectron angular distribution is altered. We present specific results for the $4 p_{1 / 2}-6 p_{1 / 2}$ and $4 p_{3 / 2}-6 p_{3 / 2}$ systems of the $\mathrm{K}$ atom and show that the change of the polarization angle leads to a significant modification of the photoelectron angular distribution.
\end{abstract}

DOI: 10.1103/PhysRevA.72.053416

PACS number(s): $32.80 . \mathrm{Qk}, 42.50 . \mathrm{Hz}, 32.80 . \mathrm{Rm}$

\section{INTRODUCTION}

The similarity between the autoionizing structure (AIS) and the laser-induced continuum structure (LICS) is very well known. In an AIS process a discrete state lying above the ionization threshold is coupled to the continuum through a configuration interaction, a resonance structure being created. In LICS two bound states are coupled to the common continuum through two laser fields (the probe and dressing lasers). The bound state coupled to the continuum through a strong laser (dressing laser) can also create a resonance structure having AIS-like properties, but compared to the AIS resonance its position and width are now controllable by the frequencies and intensities of the lasers. The first experimental observation of LICS was successfully reported in Refs. [1,2]. More comprehensive information on LICS can be found in a review paper by Knight et al. [3].

Through LICS not only the ionization yield but also a number of some other processes can be altered: Several works based on LICS investigated nonlinear optical effects such as the enhancement of third-high-harmonic generation [4]. In Ref. [5], effects of LICS on spin polarization were studied for heavy alkali-metal atoms. Recently, LICS for multiple continua was experimentally and theoretically investigated [6]. In Ref. [7], the control of ionization products in LICS was suggested for the case of decay into multiple continua.

It is well known that the photoelectron angular distribution (PAD) provides more information about the ionization process than the angle-integrated ionization signal [8]. PAD's of $\mathrm{Na}$ by the two linearly polarized lasers with a variable polarization were reported in Ref. [9], and the phase difference between the continua with same parity was extracted. By measuring PAD's of an alkali-metal atom in a bichromatic laser field, a theoretical method was proposed in Ref.

\footnotetext{
*Permanent address: Institute for Space Sciences, P.O. Box MG23, Ro 77125, Bucharest-Măgurele, Romania.

†Electronic address: t-nakajima@iae.kyoto-u.ac.jp
}

[10] in order to extract the phase difference of the continua with opposite parities.

Most recently we have theoretically investigated how LICS affects PAD [11], and specific results have been presented for the $\mathrm{K}$ atom. In Ref. [11], however, we have assumed that the probe and dressing lasers are linearly polarized along the same direction. A natural question would be how the PAD is modified, through LICS, by changing the relative polarization angle between the probe and dressing lasers.

The aim of the present paper is to generalize our previous work [11]. We now vary the relative polarization angle and analyze the modifications of the ionization yield and the photoelectron angular distribution through LICS and see how the enhancement or suppression of a particular ionization channel takes place. Since the photoelectrons ejected into different involved continua have different angular distributions and those angular distributions depend on the relative polarization angle, we expect important modifications in terms of the ionization yields, branching ratios, and photoelectron angular distributions.

The paper is organized as follows. In Sec. II we present the theoretical model: The time-dependent amplitude equations which describe the dynamics of the LICS process are derived, and the ionization yield and photoelectron angular distribution are calculated. The consistency of our results has been checked using an alternative approach based on the density matrix equations. The theoretical results obtained using these two formalisms are of course identical. Further details about the density matrix formalism are provided at the beginning of Sec. III and in Appendix A. Section III is mainly devoted to discussions of the numerical results for the total and partial ionization rates, branching ratios, and PAD's at different polarization angles. The atomic parameters needed for the LICS calculation are given in Appendices B-D.

\section{THEORY}

The system we consider in this paper consists of an initially occupied $4 p$ state, initially unoccupied $6 p$ state, and the 


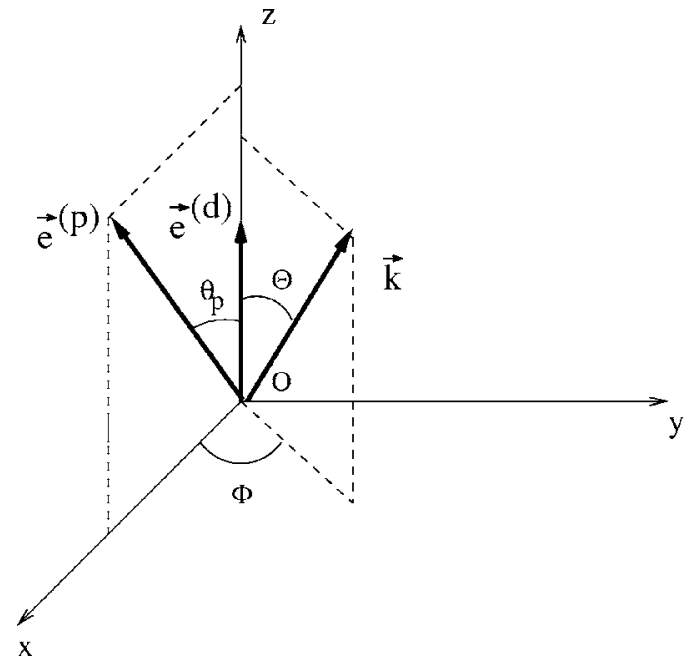

FIG. 1. Quantization axis and the polarization vectors $\mathbf{e}^{(p)}$ and $\mathbf{e}^{(d)}$ for the probe and dressing lasers defined for this work. The polarization vector $\mathbf{e}^{(p)}$ lies in the $x-z$ plane, and the quantization axis is taken along the $z$ axis.

continuum states of the $\mathrm{K}$ atom together with the linearly polarized probe and dressing lasers that couple $4 p$ and $6 p$ to the continuum states, respectively. This implies that, prior to the interaction of the system with the probe and dressing lasers, $\mathrm{K}$ atoms in the ground $4 s$ state have been excited to the $4 p$ state by a linearly polarized auxiliary laser. By choosing an appropriate frequency of the auxiliary laser, we can selectively excite either $4 p_{1 / 2}\left(m_{j}= \pm 1 / 2\right)$ or $4 p_{3 / 2}\left(m_{j}\right.$ $= \pm 1 / 2$ ), which will serve as an initial state in this work. For simplicity, we assume that the polarization axis of the dressing laser is parallel to that of the auxiliary laser, while the polarization axis of the probe laser can be arbitrary.

Here we are interested in a particular geometry where the polarization vector of the dressing and auxiliary lasers are along the $z$ axis and that of the probe laser is assumed to lie in the $x-z$ plane, as shown in Fig. 1. For such a case, the polarization vector of the probe laser is defined as $\mathbf{e}^{(p)}$ $=\mathbf{e}_{1} \sin \theta_{p}+\mathbf{e}_{3} \cos \theta_{p}$, where $\mathbf{e}_{1}$, and $\mathbf{e}_{3}$ are the unit vectors along the $x$ and $z$ axes, respectively, and $\theta_{p}$ represents the polarization angle of the probe laser with respect to that of the dressing laser. Defining the frequencies of the probe and dressing lasers as $\omega_{p}$ and $\omega_{d}$, respectively, the total electric field vector can be written as

$$
\mathbf{E}(t)=\sum_{\alpha=p, d} \mathcal{E}_{0 \alpha}(t) \mathbf{e}^{(\alpha)} \cos \left(\omega_{\alpha} t\right)
$$

A Gaussian temporal profile was employed for the amplitude of the laser fields: $\mathcal{E}_{0 \alpha}(t)=\mathcal{E}_{0 \alpha} \exp \left[-4 \ln 2\left(t / \tau_{\alpha}\right)^{2}\right]$, where $\tau_{\alpha}$ represents the temporal width for the full width at half maximum (FWHM) of the probe or dressing pulse with $\alpha=p$ or $d$, indicating the probe and dressing pulses. $\mathbf{e}^{(\alpha)}$ is the polarization vector of the laser pulse $\alpha$.

Based on the above assumptions, the level scheme we consider in this paper is now described in Figs. 2(a)-2(c) for the $\mathrm{K} 4 p_{1 / 2}-6 p_{1 / 2}$ system, at $\theta_{p}=0^{\circ}, \theta_{p}=90^{\circ}$, and in between-i.e., $0^{\circ}<\theta_{p}<90^{\circ}$. If both polarization axes of the (a)

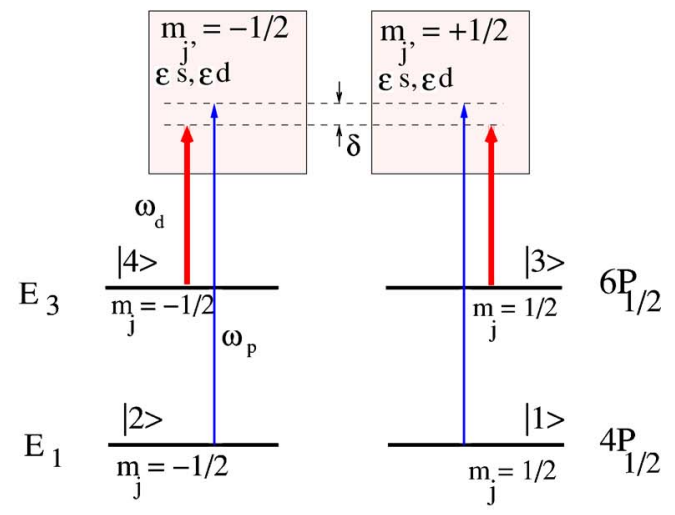

(b)

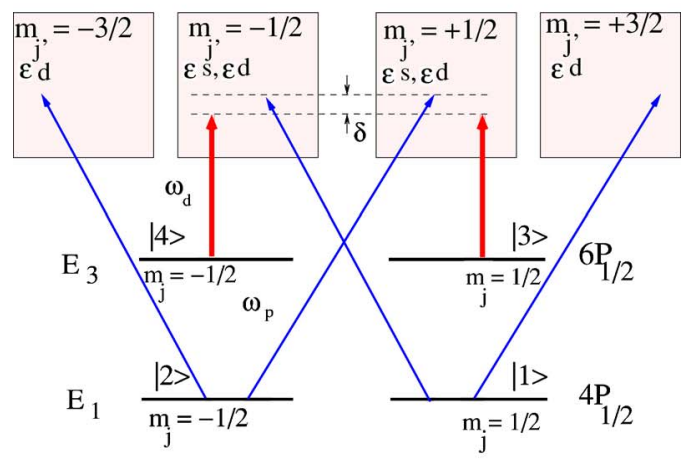

(c)

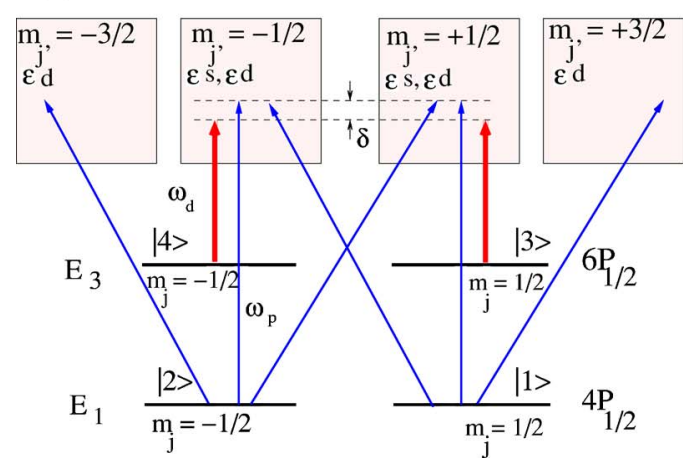

FIG. 2. (Color online) Level scheme considered in this paper for the $\mathrm{K} 4 p_{1 / 2}-6 p_{1 / 2}$ system. Depending on the polarization angle of the probe laser, $\theta_{p}$, different transition paths have to be considered. (a) $\theta_{p}=0^{\circ}$, (b) $\theta_{p}=90^{\circ}$, and (c) $0^{\circ}<\theta_{p}<90^{\circ}$. A similar level scheme can be drawn for the $\mathrm{K} 4 p_{3 / 2}-6 p_{3 / 2}$ system.

probe and dressing lasers are parallel-i.e., $\theta_{p}=0^{\circ}$ as shown in Fig. 2(a)—due to the selection rule $m_{j^{\prime}}=m_{j}$ (where the prime index is used for quantum numbers of the continuum), the entire $4 p_{1 / 2}-6 p_{1 / 2}$ system with $m_{j}= \pm 1 / 2$ can be decomposed into the two independent subsystems which consist of $4 p_{1 / 2}\left(m_{j}= \pm 1 / 2\right), 6 p_{1 / 2}\left(m_{j}= \pm 1 / 2\right)$, and the continua $\epsilon s\left(m_{j^{\prime}}\right.$ $= \pm 1 / 2)$ and $\epsilon d\left(m_{j^{\prime}}= \pm 1 / 2\right)$. The ionization yields for these subsystems are obviously symmetric to each other, and for simplicity we can consider only one of them, as already explained in our previous paper [11]. Similarly, at $\theta_{p}=90^{\circ}$, because of the selection rule $m_{j^{\prime}}=m_{j} \pm 1$, the entire $4 p_{1 / 2}-6 p_{1 / 2}$ system with $m_{j}= \pm 1 / 2$ can be decomposed into the two in- 
dependent subsystems consisting of $4 p_{1 / 2}\left(m_{j}= \pm 1 / 2\right)$, $6 p_{1 / 2}\left(m_{j}=\mp 1 / 2\right)$, and the continua $\epsilon s\left(m_{j^{\prime}}=\mp 1 / 2\right)$ and $\epsilon d\left(m_{j^{\prime}}=\mp 1 / 2\right)$ with additional incoherent channels $\epsilon d\left(m_{j^{\prime}}\right.$ $= \pm 3 / 2$ ), as shown in Fig. 2(b). Again, both subsystems are completely symmetric, and it is sufficient to study only one of the two subsystems. However, for the intermediate values of the polarization angle, $0^{\circ}<\theta_{p}<90^{\circ}$ as shown in Fig. 2 (c), because of the selection rules $m_{j^{\prime}}=m_{j} \pm 1$ (from the perpendicular component of the probe polarization vector with respect to the quantization axis, $\theta_{p}=90^{\circ}$ ) and $m_{j^{\prime}}=m_{j}$ (from the parallel component, $\theta_{p}=0^{\circ}$ ), the entire system $4 p_{1 / 2}-6 p_{1 / 2}$ cannot be decomposed into the two independent subsystems anymore, and the entire system-4 $-4 p_{1 / 2}, 6 p_{1 / 2}$, and the continua with all possible magnetic sublevels-has to be taken into account at the same time. The continuum states $\left|c_{b}\right\rangle(b=5,8)$, not presented in Fig. 2, have the same quantum numbers as the continuum states $\left|c_{a}\right\rangle(a=1,4$, for $\left.m_{j^{\prime}}=+3 / 2,+1 / 2,-1 / 2,-3 / 2\right)$, but they correspond to a different value of energy because of the incoherent one-photon ionization from $6 p_{1 / 2}$ by the probe laser. A similar level scheme, taking into account appropriate dipole selection rules, has been considered for the $\mathrm{K} 4 p_{3 / 2}-6 p_{3 / 2}$ system.

In order to observe LICS, it is necessary that the initially occupied $4 p_{1 / 2}$ (or $4 p_{3 / 2}$ ) state (denoted as $|1\rangle$ for the magnetic sublevel having $m_{j}=+1 / 2$ and $|2\rangle$ for the magnetic sublevel having $m_{j}=-1 / 2$ ) and the initially unoccupied $6 p_{1 / 2}$ (or $6 p_{3 / 2}$ ) state (denoted as $|3\rangle$ for $m_{j}=+1 / 2$ and $|4\rangle$ for $m_{j}=$ $-1 / 2$ ) be coupled by the probe and dressing lasers whose frequencies nearly satisfy the two-photon resonance-i.e., $E_{4 p}+\omega_{p} \simeq E_{6 p}+\omega_{d}$. As long as we use a ns laser with appropriate intensities and detunings, it is perfectly valid to treat each $4 p_{1 / 2}-6 p_{1 / 2}$ and $4 p_{3 / 2}-6 p_{3 / 2}$ system separately, as was explained in our previous paper [11].

It should be mentioned that we expect a different behavior of the two systems: For the $\mathrm{K} 4 p_{1 / 2}-6 p_{1 / 2}$ system the initial state $4 p_{1 / 2}$ is an isotropic mixture of all possible magnetic sublevels (recall that the magnetic sublevels $m_{j}= \pm 1 / 2$ are equally populated by the auxiliary laser), implying that the initial state is spherically symmetric. It is obvious that the PAD from the spherically symmetric initial state orientates along the polarization axis of the probe laser if the dressing laser is off. The PAD changes neither its shape nor magnitude [12]. That is not the case for the $\mathrm{K} 4 p_{3 / 2}-6 p_{3 / 2}$ system, since not all possible magnetic sublevels are excited with the same probability, and accordingly the initial state $4 p_{3 / 2}$ is nonspherical (polarized). Therefore, we expect a quite different modification of the PAD for the $4 p_{1 / 2}-6 p_{1 / 2}$ and $4 p_{3 / 2}-6 p_{3 / 2}$ systems through LICS by varying the relative polarization angle, as we have already seen for $\theta_{p}=0^{\circ}$ [11]. Further details will be provided in Sec. III.

\section{A. Time-dependent amplitude equations}

In order to study the temporal evolution of the atomic system in a laser field, we have used the standard procedure as described in our previous paper [11]. Briefly, we solve the following set of time-dependent amplitude equations:

$$
\dot{u}_{1}=-\frac{1}{2} \widetilde{\Gamma}_{1} u_{1}-i \Omega_{13}\left(1-\frac{i}{q_{13}}\right) u_{3}-i \Omega_{14}\left(1-\frac{i}{q_{14}}\right) u_{4},
$$

$$
\begin{gathered}
\dot{u}_{2}=-\frac{1}{2} \widetilde{\Gamma}_{2} u_{2}-i \Omega_{23}\left(1-\frac{i}{q_{23}}\right) u_{3}-i \Omega_{24}\left(1-\frac{i}{q_{24}}\right) u_{4}, \\
\dot{u}_{3}=\left(i \delta-\frac{1}{2} \widetilde{\Gamma}_{3}\right) u_{3}-i \Omega_{31}\left(1-\frac{i}{q_{31}}\right) u_{1}-i \Omega_{32}\left(1-\frac{i}{q_{32}}\right) u_{2}, \\
\dot{u}_{4}=\left(i \delta-\frac{1}{2} \widetilde{\Gamma}_{4}\right) u_{4}-i \Omega_{41}\left(1-\frac{i}{q_{41}}\right) u_{1}-i \Omega_{42}\left(1-\frac{i}{q_{42}}\right) u_{2},
\end{gathered}
$$

where $u_{j}$ 's $(j=1,4)$ represent the probability amplitudes of states $|j\rangle$. Note that all the probability amplitudes for the continuum states have already been adiabatically eliminated in Eqs. (2)-(5). $\delta$ is a two-photon detuning defined by $\delta$ $=\delta_{\text {static }}+\delta_{\text {stark }}$, where the static detuning is defined by $\delta_{\text {static }}$ $=\left(E_{1}+\hbar \omega_{p}\right)-\left(E_{3}+\hbar \omega_{d}\right)$, and $\delta_{\text {stark }}$ is a total dynamic ac Stark shift defined by $\delta_{\text {stark }}=\left(S_{1}^{(p)}+S_{1}^{(d)}\right)-\left(S_{3}^{(p)}+S_{3}^{(d)}\right)$. In all the numerical results presented in this work the zero point of the detuning has been chosen such that $\delta \rightarrow \delta-\delta_{\text {star }}^{\text {max }}$, since the ac Stark shifts simply translate the LICS resonance on the detuning scale. The superscript of $\delta_{\text {stark }}^{\text {max }}$ means that the ac Stark shift is calculated at the peak value of the laser intensity. $D_{j c}^{(\alpha)}$ 's are the bound-free matrix elements by the laser $\alpha(\alpha$ $=p$ or $d$ ) from the bound state $|j\rangle$ to the continuum $|c\rangle$, which are connected to the partial ionization widths through the relation $\Gamma_{j c}^{(\alpha)}=2 \pi\left|D_{j c}^{(\alpha)}\right|^{2}$. $\widetilde{\Gamma}_{j}$ represents the total ionization width of state $|j\rangle$-i.e., $\widetilde{\Gamma}_{j} \equiv \gamma_{j}+\Gamma_{j}^{(p)}$ (for $j=1,2$ ) and $\widetilde{\Gamma}_{j}$ $\equiv \gamma_{j}+\Gamma_{j}^{(d)}+\Gamma_{j}^{(p)}($ for $j=3,4)$, where $\gamma_{j}$ is the phenomenologically introduced spontaneous decay width of state $|j\rangle$. In the above equations the two-photon Rabi frequency $\Omega_{i j}$ can be written as a sum of the partial two-photon Rabi frequencies into the coherent $\epsilon s$ and $\epsilon d$ continua of energy $\epsilon$-i.e.,

$$
\Omega_{i j}\left(1-\frac{i}{q_{i j}}\right)=\sum_{\beta=\epsilon s, \epsilon d} \Omega_{i j}^{\beta}\left(1-\frac{i}{q_{i j}^{\beta}}\right),
$$

where $q_{i j}$ and $q_{i j}^{\beta}$ represent the total and partial asymmetry parameters, respectively. It is very well known that, for the light alkali metals such as $\mathrm{Li}, \mathrm{N}$, and $\mathrm{K}$, the dependence of radial matrix elements and phase shifts on the total angular momentum quantum number $j$ is very small and can be neglected [13]. Now, the following relations are satisfied by the angle-integrated atomic parameters: $\Gamma_{1}^{(\alpha)}=\Gamma_{2}^{(\alpha)}, \Gamma_{3}^{(\alpha)}=\Gamma_{4}^{(\alpha)}$, $S_{1}^{(\alpha)}=S_{2}^{(\alpha)}, S_{3}^{(\alpha)}=S_{4}^{(\alpha)}, \quad \Omega_{13}=\Omega_{24}, \quad \Omega_{14}=-\Omega_{23}, q_{13}=q_{24}$, and $q_{14}=-q_{23}$. Details about the calculation of the atomic parameters such as Rabi frequencies, ionization widths, ac Stark shifts, and asymmetry parameters are given in Appendices B-D.

Since the behavior of the population dynamics in the continuum is of our interest, we also need the following set of amplitude equations for the continua:

$$
\dot{u}_{c_{a}}=-i \delta_{c_{a}} u_{c_{a}}-i D_{c_{a} 1}^{(p)} u_{1}-i D_{c_{a}{ }^{2}}^{(p)} u_{2}-i D_{c_{a}{ }^{3}}^{(d)} u_{3}-i D_{c_{a} 4}^{(d)} u_{4},
$$




$$
\dot{u}_{c_{b}}=-i \delta_{c_{b}} u_{c_{b}}-i D_{c_{b}{ }^{3}}^{(p)} u_{3}-i D_{c_{b} 4}^{(p)} u_{4} .
$$

Here $u_{c_{a}}$ represents the probability amplitude of the coherent continuum state $\left|c_{a}\right\rangle(a=1,4)$ and $u_{c_{b}}$ represents the probability amplitude of the incoherent continuum state $\left|c_{b}\right\rangle(b$ $=5,8)$. As already explained at the beginning of Sec. II the incoherent continuum states $\left|c_{b}\right\rangle$ are not presented in Fig. 2 to avoid the complexity of the figure. They have the same quantum numbers as the coherent continuum states $\left|c_{a}\right\rangle$, but located at different energies.

Using the solutions to Eqs. (2)-(5), (7), and (8), we can now calculate the total (angle-integrated) ionization yield $R(t)$ from the relation

$$
R(t)=\sum_{a=1}^{8} R_{c_{a}}(t),
$$

where the partial photoelectron yields $R_{c}(t)$, into each coherent and incoherent continuum state $\left|c_{a}\right\rangle(a=1,8)$ can be calculated through the following formulas:

$$
\begin{gathered}
R_{c_{1}}(t)=\int_{-\infty}^{t} d t^{\prime} \Gamma_{1 c_{1}}^{(p)}\left|u_{1}\right|^{2}, \\
R_{c_{2}}(t)=\int_{-\infty}^{t} d t^{\prime}\left\{\Gamma_{1 c_{2}}^{(p)}\left|u_{1}\right|^{2}+\Gamma_{2 c_{2}}^{(p)}\left|u_{2}\right|^{2}+\Gamma_{3 c_{2}}^{(d)}\left|u_{3}\right|^{2}\right. \\
+4 \operatorname{Im}\left[\Omega_{13}^{c_{2}}\left(1+\frac{i}{q_{13}^{c_{2}}}\right)\right] \operatorname{Re}\left(u_{1} u_{3}^{*}\right) \\
\left.+4 \operatorname{Im}\left[\Omega_{23}^{c_{2}}\left(1+\frac{i}{q_{23}^{c_{2}}}\right)\right] \operatorname{Re}\left(u_{2} u_{3}^{*}\right)\right\}, \\
R_{c_{3}}(t)=\int_{-\infty}^{t} d t^{\prime}\left\{\Gamma_{1 c_{3}}^{(p)}\left|u_{1}\right|^{2}+\Gamma_{2 c_{3}}^{(p)}\left|u_{2}\right|^{2}+\Gamma_{4 c_{3}}^{(d)}\left|u_{4}\right|^{2}\right. \\
+4 \operatorname{Im}\left[\Omega_{14}^{c_{3}}\left(1+\frac{i}{q_{14}^{c_{3}}}\right)\right] \operatorname{Re}\left(u_{1} u_{4}^{*}\right) \\
\left.+4 \operatorname{Im}\left[\Omega_{24}^{c_{3}}\left(1+\frac{i}{q_{24}^{c_{3}}}\right)\right] \operatorname{Re}\left(u_{2} u_{4}^{*}\right)\right\}, \\
R_{c_{7}}(t)=\int_{-\infty}^{t} d t^{\prime}\left[\Gamma_{3 c_{7}}^{(p)}\left|u_{3}\right|^{2}+\Gamma_{4 c_{7}}^{(p)}\left|u_{4}\right|^{2}\right], \\
R_{c_{6}}(t)=\int_{-\infty}^{t} d t^{\prime}\left[\Gamma_{3 c_{6}}^{(p)}\left|u_{3}\right|^{2}+\Gamma_{4 c_{6}}^{(p)}\left|u_{4}\right|^{2}\right], \\
R_{c_{5}}(t)=\int_{-\infty}^{t} d t^{\prime} \Gamma_{2 c_{4}}^{(p)}\left|u_{2}\right|^{2}, \\
t t^{\prime} \Gamma_{3 c_{5}}^{(p)}\left|u_{3}\right|^{2},
\end{gathered}
$$

$$
R_{c_{8}}(t)=\int_{-\infty}^{t} d t^{\prime} \Gamma_{4 c_{8}}^{(p)}\left|u_{4}\right|^{2}
$$

Since the total ionization yield is a sum of ionization into the coherent and incoherent continua, it might be rewritten as

$$
\begin{aligned}
R(t) & =\sum_{\beta=\epsilon s, \epsilon d}\left[\sum_{a=1}^{4} R_{c_{a}}^{\beta}(t)+\sum_{b=5}^{8} R_{c_{b}}^{\beta}(t)\right] \\
& =\sum_{\beta=\epsilon s, \epsilon d}\left[R_{\beta}(t)+R_{\beta}^{\text {incoh }}(t)\right] .
\end{aligned}
$$

To see the effects of LICS, it is useful to calculate the branching ratio $B$, defined as the ratio between the partial ionization yield into each coherent continuum $\epsilon d$ and $\epsilon s$ :

$$
B=\frac{R_{\epsilon d}}{R_{\epsilon s}} .
$$

The total ionization yield given by Eq. (9) and the partial ionization yields given by Eqs. (10)-(17) are calculated at the end of the pulses.

\section{B. Photoelectron angular distribution}

For the purpose of calculating photoelectron angular distribution we need equations before angle integration. In order to simplify the calculation of the bound-free dipole matrix elements we use a partial-wave expansion for the continuum of an alkali-metal atom in a coupled $\left|\left(l^{\prime} s^{\prime}\right) j^{\prime} m_{j^{\prime}}\right\rangle$ basis:

$$
\begin{aligned}
\left|\mathbf{k} ; m_{s^{\prime}}\right\rangle= & \sum_{l^{\prime}, m_{l^{\prime}, j^{\prime}}} a_{l^{\prime} m_{l^{\prime}}}(-1)^{l^{\prime}-1 / 2+m_{l^{\prime}}+m_{s^{\prime}}} \sqrt{2 j^{\prime}+1} \\
& \times\left(\begin{array}{ccc}
l^{\prime} & 1 / 2 & j^{\prime} \\
m_{l^{\prime}} & m_{s^{\prime}} & -m_{j^{\prime}}
\end{array}\right)\left|\mathbf{k} ;\left(l^{\prime} s^{\prime}\right) j^{\prime} m_{j^{\prime}}\right\rangle,
\end{aligned}
$$

where $\mathbf{k}$ represents the wave vector of photoelectron, $a_{l^{\prime} m_{l^{\prime}}}$

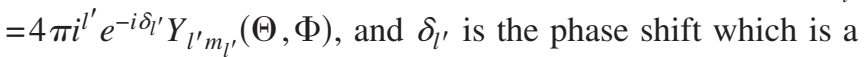
sum of the Coulomb phase shift and the scattering phase shift; recall that a prime indicates a quantum number for the continuum state.

We are interested in the PAD as a function of polarization angle $\theta_{p}$. If the final spin state of the photoelectron is not detected, we have to incoherently sum over the final spin projection $m_{s^{\prime}}$. The partial photoelectron yield into a solid angle $\Omega_{\mathbf{k}}$, defined by the polar angle $\Theta$, and the azimuthal angle $\Phi$, can be written as

$$
\begin{aligned}
\left.\frac{d R(\Theta, \Phi)}{d t d \Omega_{\mathbf{k}}}\right|_{m_{j}= \pm 1 / 2}= & 0.589 \pi \sum_{m_{s^{\prime}}= \pm 1 / 2}\left[\mid \sum_{j=1}^{2} \sqrt{\Gamma_{j}^{\left(p, m_{s^{\prime}}\right)}(\Theta, \Phi)} u_{j}\right. \\
& +\left.\sum_{j=3}^{4} \sqrt{\Gamma_{j}^{\left(d, m_{s^{\prime}}\right)}(\Theta, \Phi)} u_{j}\right|^{2} \\
& \left.+\left|\sum_{j=3}^{4} \sqrt{\Gamma_{j}^{\left(p, m_{s^{\prime}}\right)}(\Theta, \Phi)} u_{j}\right|^{2}\right],
\end{aligned}
$$

where $0.589 \pi$ is a conversion factor for the appropriate normalization and the formula is valid for both $4 p_{1 / 2}-6 p_{1 / 2}$ and 
$4 p_{3 / 2}-6 p_{3 / 2}$ systems for $m_{j}= \pm 1 / 2$ of the initial state. After considerable angular momentum algebra, we obtain expressions for the differential ionization widths $\Gamma_{i}^{\left(\alpha, m_{s^{\prime}}\right)}(\Theta, \Phi)$ from states $|1\rangle$ and $|3\rangle$ for the $4 p_{1 / 2}-6 p_{1 / 2}$ system,

$$
\begin{aligned}
& \Gamma_{j}^{(\alpha,+1 / 2)}(\Theta, \Phi)=\mid-\frac{1}{3} R_{j \in s}^{(\alpha)} e^{i \delta_{s}} Y_{00}(\Theta, \Phi) e_{0}^{(\alpha)} \\
& +\frac{2}{3 \sqrt{5}} R_{j \in d}^{(\alpha)} e^{i \delta_{d}} Y_{20}(\Theta, \Phi) e_{0}^{(\alpha)} \\
& +\frac{1}{\sqrt{15}} R_{j \epsilon d}^{(\alpha)} e^{i \delta_{d}} Y_{21}(\Theta, \Phi) e_{1}^{(\alpha)} \\
& +\left.\frac{1}{\sqrt{15}} R_{j \in d}^{(\alpha)} e^{i \delta_{d}} Y_{2-1}(\Theta, \Phi) e_{-1}^{(\alpha)}\right|^{2} I_{\alpha}, \\
& \Gamma_{j}^{(\alpha,-1 / 2)}(\Theta, \Phi)=\mid-\frac{\sqrt{2}}{3} R_{j \in s}^{(\alpha)} e^{i \delta_{s}} Y_{00}(\Theta, \Phi) e_{-1}^{(\alpha)} \\
& -\frac{\sqrt{2}}{3 \sqrt{5}} R_{j \epsilon d}^{(\alpha)} e^{i \delta_{d}} Y_{20}(\Theta, \Phi) e_{-1}^{(\alpha)} \\
& -\frac{2}{\sqrt{15}} R_{j \in d}^{(\alpha)} d^{i \delta_{d}} Y_{22}(\Theta, \Phi) e_{1}^{(\alpha)} \\
& -\left.\frac{\sqrt{2}}{\sqrt{15}} R_{j \in d}^{(\alpha)} e^{i \delta_{d}} Y_{21}(\Theta, \Phi) e_{0}^{(\alpha)}\right|^{2} I_{\alpha},
\end{aligned}
$$

and for the differential ionization widths $\Gamma_{j}^{\left(\alpha, m_{s^{\prime}}\right)}(\Theta, \Phi)$, from states $|2\rangle$ and $|4\rangle$,

$$
\begin{aligned}
\Gamma_{j}^{(\alpha,+1 / 2)}(\Theta, \Phi)= & \frac{\sqrt{2}}{3} R_{j \epsilon s}^{(\alpha)} e^{i \delta_{s}} Y_{00}(\Theta, \Phi) e_{1}^{(\alpha)} \\
& +\frac{\sqrt{2}}{3 \sqrt{5}} R_{j \epsilon d}^{(\alpha)} e^{i \delta_{d} Y_{20}(\Theta, \Phi) e_{1}^{(\alpha)}} \\
& +\frac{\sqrt{2}}{\sqrt{15}} R_{j \epsilon d}^{(\alpha)} e^{i \delta_{d} Y_{2-1}(\Theta, \Phi) e_{0}^{(\alpha)}} \\
& +\left.\frac{2}{\sqrt{15}} R_{j \epsilon d}^{(\alpha)} e^{i \delta_{d}} Y_{2-2}(\Theta, \Phi) e_{-1}^{(\alpha)}\right|^{2} I_{\alpha}, \\
\Gamma_{j}^{(\alpha,-1 / 2)}(\Theta, \Phi)= & \mid \frac{1}{3} R_{j \epsilon s}^{(\alpha)} e^{i \delta_{s}} Y_{00}(\Theta, \Phi) e_{0}^{(\alpha)} \\
& -\frac{2}{3 \sqrt{5}} R_{j \in d}^{(\alpha)} e^{i \delta_{d}} Y_{20}(\Theta, \Phi) e_{0}^{(\alpha)} \\
& -\frac{1}{\sqrt{15}} R_{j \epsilon d}^{(\alpha)} e^{i \delta_{d}} Y_{21}(\Theta, \Phi) e_{1}^{(\alpha)} \\
& -\left.\frac{1}{\sqrt{15}} R_{j \epsilon d}^{(\alpha)} e^{i \delta_{d}} Y_{2-1}(\Theta, \Phi) e_{-1}^{(\alpha)}\right|^{2} I_{\alpha},
\end{aligned}
$$

where $R_{j \epsilon s}^{(\alpha)}$ and $R_{j \in d}^{(\alpha)}$ represent the radial bound-free matrix elements from state $|j\rangle(j=1,4)$ to the continua $\epsilon s$ and $\epsilon d$, respectively, by laser $\alpha(\alpha=p$ or $d)$, evaluated in atomic units. Here $e_{q}^{(\alpha)}$, with $q=0, \pm 1$, are the spherical components of the polarization vector of laser $\alpha$-namely, $e_{0}^{(\alpha)}=\cos \theta_{\alpha}$ and $e_{ \pm 1}^{(\alpha)}=\mp \sin \theta_{\alpha} / \sqrt{2}$. The laser intensities $I_{p}$ and $I_{d}$ are expressed in $\mathrm{W} / \mathrm{cm}^{2}$. For the coherent continuum, the relevant phase shifts are $\delta_{s}=1.937$ and $\delta_{d}=-6.574$, which are the sum of the Coulomb phase shifts, $\delta_{s}^{C}=-4.924$ and $\delta_{d}^{C}=-7.551$, and the scattering phase shifts, $\pi \mu_{s}=6.861$ and $\pi \mu_{d}=0.977$, with $\mu_{l}(l=s, d)$ being the quantum defects estimated from the linear extrapolation of the bound Rydberg $s$ and $d$ series of the $\mathrm{K}$ atom to the continuum energy of interest. Equation (21) together with Eqs. (22)-(25) gives the PAD for the $4 p_{1 / 2}-6 p_{1 / 2}$ system with appropriate normalization, so that the angle-integrated quantity becomes identical to the total ionization yield calculated with Eq. (9).

Similarly the differential ionization widths from states $|1\rangle$ and $|3\rangle$ for the $4 p_{3 / 2}-6 p_{3 / 2}$ system are given by

$$
\begin{aligned}
\Gamma_{j}^{(\alpha,+1 / 2)}(\Theta, \Phi)= & \frac{\sqrt{2}}{3} R_{j \epsilon s}^{(\alpha)} e^{i \delta_{s}} Y_{00}(\Theta, \Phi) e_{0}^{(\alpha)} \\
& -\frac{2 \sqrt{2}}{3 \sqrt{5}} R_{j \epsilon d}^{(\alpha)} e^{i \delta_{d} Y_{20}(\Theta, \Phi) e_{0}^{(\alpha)}} \\
& -\frac{\sqrt{2}}{\sqrt{15}} R_{j \epsilon d}^{(\alpha)} e^{i \delta_{d} Y_{21}(\Theta, \Phi) e_{1}^{(\alpha)}} \\
& -\frac{\sqrt{2}}{\sqrt{15}} R_{j \epsilon d}^{(\alpha)} e^{\left.i \delta_{d} Y_{2-1}(\Theta, \Phi) e_{-1}^{(\alpha)}\right|^{2} I_{\alpha},}
\end{aligned}
$$

$$
\begin{aligned}
\Gamma_{j}^{(\alpha,-1 / 2)}(\Theta, \Phi)= & -\frac{1}{3} R_{j \in s}^{(\alpha)} e^{i \delta_{s}} Y_{00}(\Theta, \Phi) e_{-1}^{(\alpha)} \\
& -\frac{1}{3 \sqrt{5}} R_{j \in d}^{(\alpha)} e^{i \delta_{d}} Y_{20}(\Theta, \Phi) e_{-1}^{(\alpha)} \\
& -\frac{\sqrt{2}}{\sqrt{15}} R_{j \in d}^{(\alpha)} e^{i \delta_{d}} Y_{22}(\Theta, \Phi) e_{1}^{(\alpha)} \\
& -\frac{1}{\sqrt{15}} R_{j \in d}^{(\alpha)} e^{\left.i \delta_{d} Y_{21}(\Theta, \Phi) e_{0}^{(\alpha)}\right|^{2} I_{\alpha},}
\end{aligned}
$$

and from states $|2\rangle$ and $|4\rangle$ they are derived as

$$
\begin{aligned}
\Gamma_{j}^{(\alpha,+1 / 2)}(\Theta, \Phi)= & -\frac{1}{3} R_{j \epsilon s}^{(\alpha)} e^{i \delta_{s}} Y_{00}(\Theta, \Phi) e_{1}^{(\alpha)} \\
& -\frac{1}{3 \sqrt{5}} R_{j \epsilon d}^{(\alpha)} e^{i \delta_{d}} Y_{20}(\Theta, \Phi) e_{1}^{(\alpha)} \\
& -\frac{1}{\sqrt{15}} R_{j \epsilon d}^{(\alpha)} e^{i \delta_{d}} Y_{2-1}(\Theta, \Phi) e_{0}^{(\alpha)} \\
& -\left.\frac{\sqrt{2}}{\sqrt{15}} R_{j \epsilon d}^{(\alpha)} e^{i \delta_{d} Y_{2-2}(\Theta, \Phi) e_{-1}^{(\alpha)}}\right|^{2} I_{\alpha},
\end{aligned}
$$


TABLE I. Atomic parameters for the $\mathrm{K} 4 P_{1 / 2}-6 p_{1 / 2}$ system. $\Omega$ is measured in $\mathrm{rad} / \mathrm{s}, \Gamma$ in $\mathrm{s}^{-1}, S$ in $\mathrm{rad} / \mathrm{s}$, and $I_{d}$ in $\mathrm{W} / \mathrm{cm}^{2}$.

\begin{tabular}{lccc}
\hline \hline$\Omega_{13}$ & $-8.12 \sqrt{I_{p} I_{d}} \cos \theta_{p}$ & $q_{13}$ & -0.91 \\
$\Omega_{13}^{\epsilon s}$ & $3.47 \sqrt{I_{p} I_{d}} \cos \theta_{p}$ & $q_{13}^{\epsilon s}$ & 1.71 \\
$\Omega_{13}^{\epsilon d}$ & $-11.58 \sqrt{I_{p} I_{d}} \cos \theta_{p}$ & $q_{13}^{\epsilon d}$ & -1.69 \\
$\Omega_{14}$ & $-9.26 \sqrt{I_{p} I_{d}} \sin \theta_{p}$ & $q_{14}$ & -6.59 \\
$\Omega_{14}^{\epsilon s}$ & $3.47 \sqrt{I_{p} I_{d}} \sin \theta_{p}$ & $q_{14}^{\epsilon s}$ & 1.71 \\
$\Omega_{14}^{\epsilon d}$ & $-5.79 \sqrt{I_{p} I_{d}} \sin \theta_{p}$ & $q_{14}^{\epsilon d}$ & -1.69 \\
$\Gamma_{1}^{(p)}$ & $11.59 I_{p}$ & $S_{1}^{(p)}$ & $14.1 I_{p}$ \\
$\Gamma_{3}^{(d)}$ & $28.04 I_{d}$ & $S_{1}^{(d)}$ & $947.5 I_{d}$ \\
$\Gamma_{3}^{(p)}$ & $3.66 I_{p}$ & $S_{3}^{(p)}$ & $21.04 I_{p}$ \\
& & $S_{3}^{(d)}$ & $86.9 I_{d}$ \\
\hline \hline
\end{tabular}

$$
\begin{aligned}
\Gamma_{j}^{(\alpha,-1 / 2)}(\Theta, \Phi)= & \frac{\sqrt{2}}{3} R_{j \epsilon s}^{(\alpha)} e^{i \delta_{s}} Y_{00}(\Theta, \Phi) e_{0}^{(\alpha)} \\
& -\frac{2 \sqrt{2}}{3 \sqrt{5}} R_{j \epsilon d}^{(\alpha)} e^{i \delta_{d} Y_{20}(\Theta, \Phi) e_{0}^{(\alpha)}} \\
& -\frac{\sqrt{2}}{\sqrt{15}} R_{j \epsilon d}^{(\alpha)} e^{i \delta_{d} Y_{21}(\Theta, \Phi) e_{1}^{(\alpha)}} \\
& -\frac{\sqrt{2}}{\sqrt{15}} R_{j \epsilon d}^{(\alpha)} e^{\left.i \delta_{d} Y_{2-1}(\Theta, \Phi) e_{-1}^{(\alpha)}\right|^{2} I_{\alpha} .}
\end{aligned}
$$

Equations (22)-(29) are applicable for both probe and dressing lasers. Recalling that the polarization vector of the dressing laser is parallel to the quantization axis $\left(\theta_{d}=0^{\circ}\right)$, the relative polarization angle between the probe and dressing lasers becomes identical to $\theta_{p}$. Because of the symmetry properties of spherical harmonics, we can show that $\Gamma_{1}^{\left(\alpha, m_{s^{\prime}}\right)}$ and $\Gamma_{3}^{\left(\alpha, m_{s^{\prime}}\right)}$ are equal to $\Gamma_{2}^{\left(\alpha,-m_{s^{\prime}}\right)}$ and $\Gamma_{4}^{\left(\alpha,-m_{s^{\prime}}\right)}$, respectively, by interchanging $e_{q}^{(\alpha)}$ and $e_{-q}^{(\alpha)}$.

\section{NUMERICAL RESULTS AND DISCUSSION}

In this section we present numerical results and discussions. All the necessary single- and effective two-photon di- pole matrix elements needed for our schemes have been obtained using quantum defect theory and the Green's function technique. The calculated atomic parameters such as Rabi frequencies, asymmetry parameters, and ac Stark shifts, for the $4 p_{1 / 2}-6 p_{1 / 2}$ and $4 p_{3 / 2}-6 p_{3 / 2}$ systems, are listed in Tables I and II, respectively.

For the $\mathrm{K} 4 p_{1 / 2}-6 p_{1 / 2}$ system only Rabi frequencies are $\theta_{p}$ dependent, while all other atomic parameters such as ionization widths and ac Stark shifts by the probe laser do not present any $\theta_{p}$ dependence, since the initial $4 p_{1 / 2}$ state is isotropic $[12,14]$ in that all possible magnetic sublevels $4 p_{1 / 2}\left(m_{j}= \pm 1 / 2\right)$ are equally populated by the linearly polarized auxiliary laser. In contrast, for the $\mathrm{K} 4 p_{3 / 2}-6 p_{3 / 2}$ system the atomic parameters such as Rabi frequencies, ionization widths, and ac Stark shifts by the probe laser depend on the polarization angle $\theta_{p}$. This is due to the fact that all the magnetic sublevels of the initial state $4 p_{3 / 2}\left(m_{j}\right.$ $= \pm 1 / 2, \pm 3 / 2)$ are not equally excited. Actually only $4 p_{3 / 2}\left(m_{j}= \pm 1 / 2\right)$ are equally excited by the linearly polarized auxiliary laser while $4 p_{3 / 2}\left(m_{j}= \pm 3 / 2\right)$ remain empty. In other words the $4 p_{1 / 2}$ state is isotropic while the $4 p_{3 / 2}$ state is aligned because of the way these states are prepared. The total and partial asymmetry parameters are independent of the laser fields [3] and, obviously, do not depend on the polarization angle of the probe laser for both $\mathrm{K} 4 p_{1 / 2}-6 p_{1 / 2}$ and $4 p_{3 / 2}-6 p_{3 / 2}$ systems.

When we solve the set of amplitude equations special care has to be taken: Note that the probability amplitudes of the initially occupied states $|1\rangle$ and $|2\rangle$ have arbitrary phases. However, if we use the amplitude equations, the initial conditions are given by the set of $u_{i}(t=-\infty)$ for all possible $i(=1,2,3,4)$ which inevitably implies that the initial coherence defined by $u_{i} u_{j}^{*}$ also exists between states with nonzero $u_{i}(t=-\infty)$ and $u_{j}(t=-\infty)$ with $i \neq j$. Of course this kind of problem does not happen for the density matrix equations at the expense of much more complicated expressions for the ionization yield, etc. Therefore, as long as we employ the amplitude equations, we have to avoid any coherent interference between the ionization paths starting from $|1\rangle$ and $|2\rangle$ because this is not physical. Therefore, we should separately solve the set of amplitude equations (2)-(5) with either $u_{1}(t=-\infty)=1$ and $u_{i}(t=-\infty)=0$ (for $\left.i=2,3,4\right)$ or

TABLE II. Atomic parameters for the $\mathrm{K} 4 P_{3 / 2}-6 p_{3 / 2}$ system. $\Omega$ is measured in $\mathrm{rad} / \mathrm{s}, \Gamma$ in $\mathrm{s}^{-1}, S$ in $\mathrm{rad} / \mathrm{s}$, and $I_{d}$ in $\mathrm{W} / \mathrm{cm}^{2}$.

\begin{tabular}{lccc}
\hline \hline$\Omega_{13}$ & $-5.80 \sqrt{I_{p} I_{d}} \cos \theta_{p}$ & $q_{13}$ & -0.5 \\
$\Omega_{13}^{\epsilon s}$ & $6.94 \sqrt{I_{p} I_{d}} \cos \theta_{p}$ & $q_{13}^{\epsilon s}$ & 1.71 \\
$\Omega_{13}^{\epsilon d}$ & $-12.74 \sqrt{I_{p} I_{d}} \cos \theta_{p}$ & $q_{13}^{\epsilon d}$ & -1.69 \\
$\Omega_{14}$ & $-9.26 \sqrt{I_{p} I_{d}} \sin \theta_{p}$ & $q_{14}$ & -6.59 \\
$\Omega_{14}^{\epsilon s}$ & $3.47 \sqrt{I_{p} I_{d}} \sin \theta_{p}$ & $q_{14}^{\epsilon s}$ & 1.71 \\
$\Omega_{14}^{\epsilon d}$ & $-5.79 \sqrt{I_{p} I_{d}} \sin \theta_{p}$ & $q_{14}^{\epsilon d}$ & -1.69 \\
$\Gamma_{1}^{(p)}$ & $\left(14.46 \cos ^{2} \theta_{p}+10.15 \sin ^{2} \theta_{p}\right) I_{p}$ & $S_{1}^{(p)}$ & $\left(12.3 \cos ^{2} \theta_{p}+15.03 \sin ^{2} \theta_{p}\right) I_{p}$ \\
$\Gamma_{3}^{(d)}$ & $38.57 I_{d}$ & $S_{1}^{(d)}$ & $1231.8 I_{d}$ \\
$\Gamma_{3}^{(p)}$ & $\left(4.32 \cos ^{2} \theta_{p}+3.33 \sin ^{2} \theta_{p}\right) I_{p}$ & $S_{3}^{(p)}$ & $\left(20.8 \cos ^{2} \theta_{p}+21.15 \sin ^{2} \theta_{p}\right) I_{p}$ \\
& & $S_{3}^{(d)}$ & $97.0 I_{d}$ \\
\hline \hline
\end{tabular}



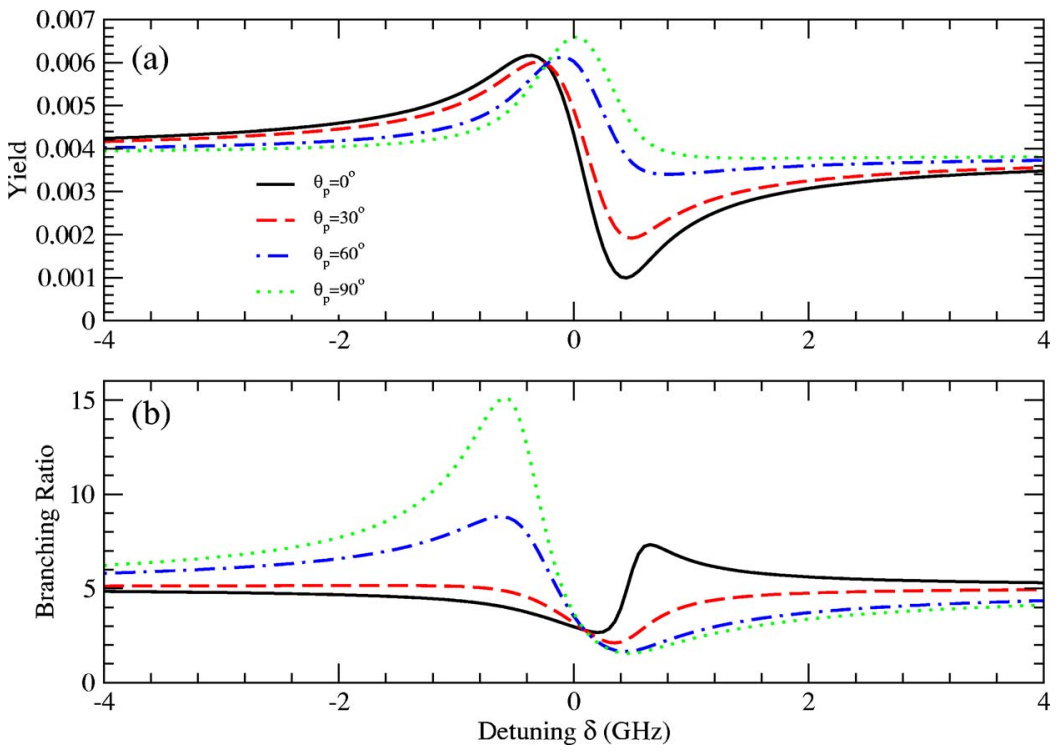

FIG. 3. (Color online) (a) Total ionization yield and (b) the branching ratio between the partial ionization yields into each $\epsilon s$ and $\epsilon d$ continuum for the $\mathrm{K} 4 p_{1 / 2}-6 p_{1 / 2}$ system as a function of two-photon detuning $\delta$. Pulse durations and peak laser intensities are chosen to be $\tau_{p}=1 \mathrm{~ns}$ and $I_{p}=1 \mathrm{MW} / \mathrm{cm}^{2}$ and $\tau_{d}=10 \mathrm{~ns}$ and $I_{d}$ $=100 \mathrm{MW} / \mathrm{cm}^{2}$ for the probe and dressing lasers, respectively. The polarization angle takes the values of $\theta_{p}=0^{\circ}, 30^{\circ}, 60^{\circ}$, and $90^{\circ}$. $u_{2}(t=-\infty)=1$ and $u_{i}(t=-\infty)=0$ (for $\left.i=1,3,4\right)$, and average the photoelectron angular distribution given by Eq. (21) over $m_{j}$ of the initial state:

$$
\frac{d R(\Theta, \Phi)}{d t d \Omega_{\mathbf{k}}}=\frac{1}{2}\left[\left.\frac{d R(\Theta, \Phi)}{d t d \Omega_{\mathbf{k}}}\right|_{m_{j}=+1 / 2}+\left.\frac{d R(\Theta, \Phi)}{d t d \Omega_{\mathbf{k}}}\right|_{m_{j}=-1 / 2}\right]
$$

Pulse durations and peak laser intensities are chosen to be $\tau_{p}=1 \mathrm{~ns}(\mathrm{FWHM})$ and $I_{p}=1 \mathrm{MW} / \mathrm{cm}^{2}$ for the probe laser and $10 \mathrm{~ns} \leqslant \tau_{d} \leqslant 15 \mathrm{~ns}$ (FWHM) and $100 \mathrm{MW} / \mathrm{cm}^{2} \leqslant I_{d}$ $\leqslant 500 \mathrm{MW} / \mathrm{cm}^{2}$ for the dressing laser. If the probe and dressing pulse durations are comparable, the LICS resonance profile is going to be smeared out [11] due to the ac Stark shifts. In order to circumvent this problem the pulse duration of the dressing laser was chosen to be much longer than that of the probe laser, since, under the condition that $\tau_{d} \gg \tau_{p}$, atomic states are quasi statistically Stark shifted by the strong dressing pulse during the interaction with the probe pulse. By substituting the atomic parameters listed in Tables I and II into Eqs. (2)-(5), we can easily solve those equations for the given peak intensities, detunings, and temporal profile of the lasers. Once the solution is obtained for $u_{i}(t)(i$ $=1,4)$, the total and partial ionization yields can be calculated from Eq. (9) and Eqs. (10)-(17). The radiative lifetimes of $4 p_{1 / 2}$ and $4 p_{3 / 2}$ and $6 p_{1 / 2}$ and $6 p_{3 / 2}$ levels are about $26 \mathrm{~ns}$ and $345 \mathrm{~ns}$, respectively, and are included in the numerical calculations.

In order to check the consistency of our results an alternative formalism based on the density matrix equations was used to calculate the dynamics of the system. The amplitude equation approach has the advantages of dealing with a fewer number of differential equations and obtaining a more compact formula for the ionization yield. On the other hand, the density matrix equations approach has the advantage of its capability to control the coherence through the offdiagonal density matrix elements (which are proportional to $\rho_{i j}=u_{i} u_{j}^{*}$, with $i \neq j$ ), and therefore it is suitably used for mixed states [such as $4 p_{1 / 2}\left(m_{j}= \pm 1 / 2\right)$ or $\left.4 p_{3 / 2}\left(m_{j}= \pm 1 / 2\right)\right]$ when at least two levels with arbitrary phase are initially occupied. Details about the density matrix approach are given in Appendix A, and the numerical results are, of course, identical to the ones obtained by using the amplitude equations. In the following subsections we present numerical results for the $\mathrm{K} 4 p_{1 / 2}-6 p_{1 / 2}$ and $4 p_{3 / 2}-6 p_{3 / 2}$ systems.

\section{A. K $4 p_{1 / 2}-6 p_{1 / 2}$ system}

Figures 3(a) and 3(b) show the variation of the total ionization yield and branching ratio as a function of two-photon detuning, $\delta$, at four different values of the polarization angle, $\theta_{p}=0^{\circ}, 30^{\circ}, 60^{\circ}$, and $90^{\circ}$. Note that the polarization angle of the dressing laser is fixed to $\theta_{d}=0^{\circ}$. Pulse durations and peak laser intensities are chosen to be $\tau_{p}=1 \mathrm{~ns}$ and $I_{p}$ $=1 \mathrm{MW} / \mathrm{cm}^{2}$ and $\tau_{d}=10 \mathrm{~ns}$ and $I_{d}=100 \mathrm{MW} / \mathrm{cm}^{2}$, for the probe and dressing lasers, respectively. Clearly, the profile of the LICS resonance and the branching ratio as a function of detuning for the $4 p_{1 / 2}-6 p_{1 / 2}$ system changes by varying the polarization angle. The position of the LICS resonance is also altered by varying $\theta_{p}$; specifically, the maximum of the LICS profile shifts toward larger values of the detuning as $\theta_{p}$ increases and its minimum vanishes completely when $\theta_{p}$ $=90^{\circ}$. At $\theta_{p}=90^{\circ}$ the value of the asymmetry parameter, which is connected to the resonance profile, $q=-6.59$, is much larger compared to the case when both lasers are linearly polarized in the same direction and the asymmetry parameter takes the value, $q=-0.91$. That particular value of the asymmetry parameter for $\theta_{p}=90^{\circ}$ is due to the fact that the corresponding angular coefficients for the $s$ and $d$ ionization channels are equal and the radial matrix elements have opposite signs.

Figures 4(a) and 4(b) show the variation of the total ionization yield and branching ratio as a function of detuning at three different dressing laser intensities $I_{d}=100,200$, and $500 \mathrm{MW} / \mathrm{cm}^{2}$, with the probe laser intensity and the pulse durations fixed to be $I_{p}=100 \mathrm{MW} / \mathrm{cm}^{2}, \tau_{p}=1 \mathrm{~ns}$, and $\tau_{d}$ $=15 \mathrm{~ns}$. The polarization angle is $\theta_{p}=30^{\circ}$. As we have already seen in Figs. 3(a) and 3(b), the ionization yields and 


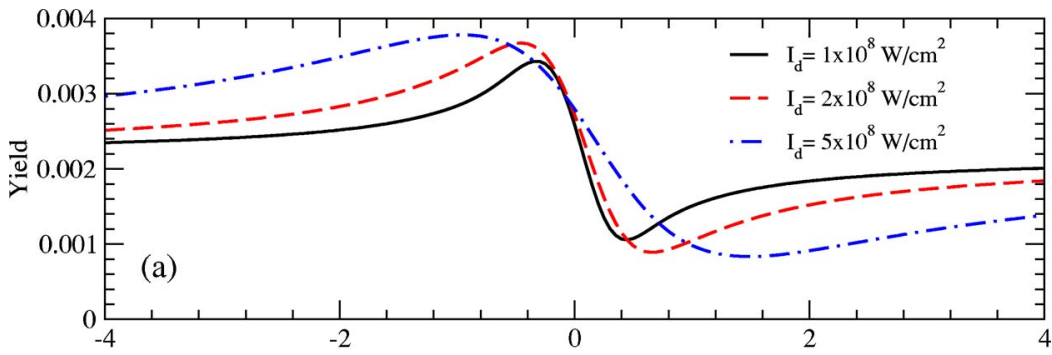

FIG. 4. (Color online) (a) Total ionization yield and (b) the branching ratio between the partial ionization yields into each $\epsilon s$ and $\epsilon d$ continuum for the $\mathrm{K} 4 p_{1 / 2}-6 p_{1 / 2}$ system as a function of two-photon detuning $\delta$ for the three different

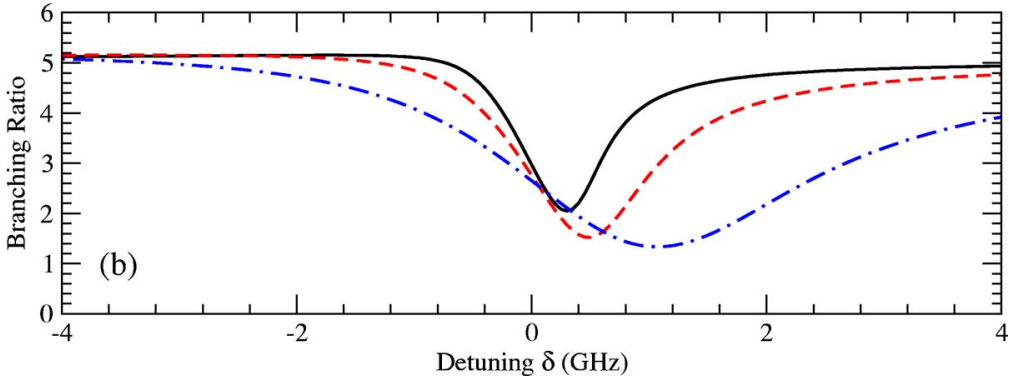
dressing laser intensities, $I_{d}=100,200$, and $500 \mathrm{MW} / \mathrm{cm}^{2}$. The intensity of the probe laser is $I_{d}=1 \mathrm{MW} / \mathrm{cm}^{2}$. Pulse durations are chosen to be $\tau_{p}=1 \mathrm{~ns}$ and $\tau_{d}=15 \mathrm{~ns}$ for the probe and dressing lasers, respectively. The polarization angle is $\theta_{p}$ $=30^{\circ}$.

branching ratios vary significantly near resonance. The LICS structure is naturally broadened as the dressing laser intensity is increased.

For the particular polarization geometry shown in Fig. 1 the azimuthal angle dependence $\Phi$ of the photoelectron signal, through the spherical harmonics $Y_{l m}(\Phi, \Theta)$, does not

(a)

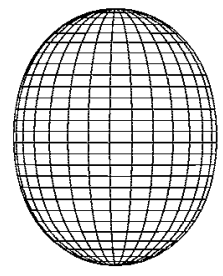

(b)

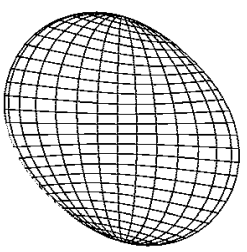

(c)

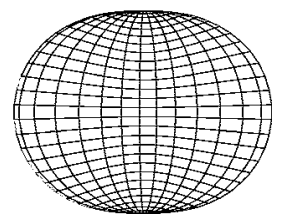

FIG. 5. Three-dimensional photoelectron angular distribution due to one-photon ionization from the $\mathrm{K} 4 p_{1 / 2}$ state by the probe laser field only at three different polarization angles $\theta_{p}=0^{\circ}, 45^{\circ}$, and $90^{\circ}$. Pulse duration and peak intensity are $\tau_{p}=1 \mathrm{~ns}$ and $I_{p}$ $=1 \mathrm{MW} / \mathrm{cm}^{2}$ for the probe laser. The view point is from the positive $y$ axis. vanish as happens when the polarization axes of both lasers are parallel to each other [11], and the cylindrical symmetry of the PAD is broken. This is due to the presence of the spherical harmonics with $m \neq 0$ in the differential ionization widths formulas, Eqs. (22)-(29). Before studying the PAD for LICS it would be instructive to give an answer to the following question: What is the modification of the PAD for one-photon ionization from the initial state $4 p_{1 / 2}$ through variation of the polarization angle of the probe laser without the dressing laser-i.e., $I_{d}=0$ ? Since the initial state $4 p_{1 / 2}$ is spherically symmetric (recall that both $m_{j}= \pm 1 / 2$ sublevels are equally populated), one could intuitively guess that the

(a)

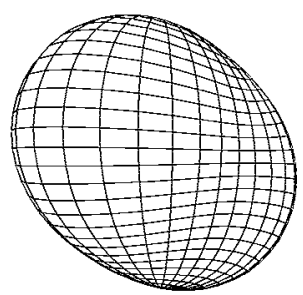

(b)

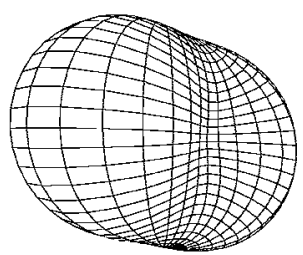

(c)

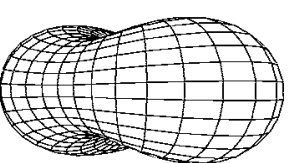

FIG. 6. Three-dimensional photoelectron angular distribution for the $\mathrm{K} 4 p_{1 / 2}-6 p_{1 / 2}$ system at three different two-photon detunings $\delta=-4,-0.62$, and $0.44 \mathrm{GHz}$. Pulse durations and peak intensities are $\tau_{p}=1 \mathrm{~ns}$ and $I_{p}=1 \mathrm{MW} / \mathrm{cm}^{2}$ for the probe laser and $\tau_{d}=10 \mathrm{~ns}$ and $I_{p}=100 \mathrm{MW} / \mathrm{cm}^{2}$ for the dressing laser. The polarization angle is $\theta_{p}=60^{\circ}$. 

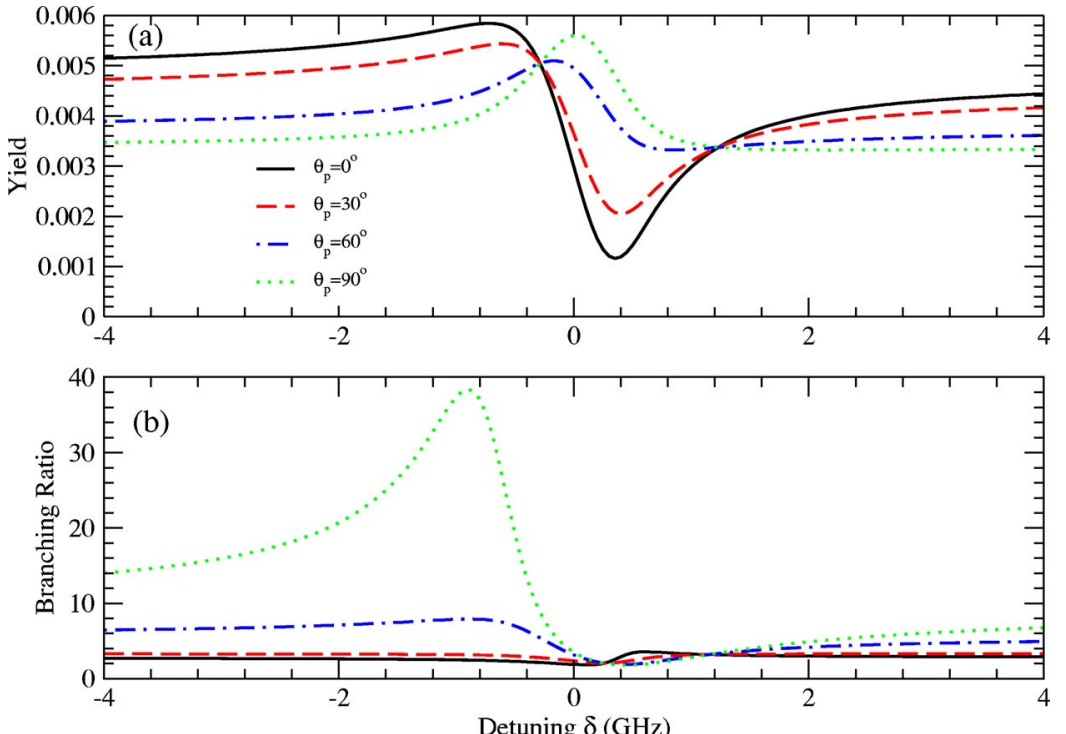

FIG. 7. (Color online) (a) Total ionization yield and (b) the branching ratio between the partial ionization yields into each $\epsilon s$ and $\epsilon d$ continuum for the $\mathrm{K} 4 p_{3 / 2}-6 p_{3 / 2}$ system as a function of two-photon detuning $\delta$. Pulse durations and peak laser intensities are chosen to be $\tau_{p}=1 \mathrm{~ns}$ and $I_{p}=1 \mathrm{MW} / \mathrm{cm}^{2}$ and $\tau_{d}=10 \mathrm{~ns}$ and $I_{d}$ $=100 \mathrm{MW} / \mathrm{cm}^{2}$ for the probe and dressing lasers, respectively. The polarization angle takes the values $\theta_{p}=0^{\circ}, 30^{\circ}, 60^{\circ}$, and $90^{\circ}$. magnitude of the PAD does not change and the PAD just aligns along the polarization axis of the probe laser. Under the condition of $I_{d}=0$, a three-dimensional (3D) PAD is plotted in Figs. 5(a)-5(c) as a function of photoelectron angles $\Theta$ and $\Phi$, for three different values of the polarization angle, $\theta_{p}=0^{\circ}, 45^{\circ}$, and $90^{\circ}$. As expected, the PAD changes its orientation along the polarization direction of the probe laser. The fourfold rotational symmetry $(\Phi \rightarrow \pi+\Phi$ and $\Phi \rightarrow-\Phi$ at $\left.\Theta=90^{\circ}\right)$, which exists when both lasers are linearly polarized along the quantization axis, breaks into a twofold symmetry $(\Phi \rightarrow \pi+\Phi)$ when the polarization of the probe laser varies $[15,16]$.

Now we consider the case of LICS; i.e., the dressing laser is turned on. Three-dimensional PAD's of the $\mathrm{K} 4 p_{1 / 2}-6 p_{1 / 2}$ system at $\theta_{p}=60^{\circ}$ are shown in Figs. 6(a)-6(c) for the three representative detunings corresponding to the far-off resonance $(\delta=-4 \mathrm{GHz})$, maximum $(\delta=-0.62 \mathrm{GHz})$, and minimum $(\delta=0.44 \mathrm{GHz})$ of the branching ratio [see Fig. 3(b)]. The viewpoint of all 3D plots in this paper is from the $x-y$ plane with the Cartesian coordinates $(2,2,0)$, if not otherwise stated. At far-off resonance [Fig. 6(a)], the 3D PAD again tends to follow the change of the polarization angle, $\theta_{p}$ $=60^{\circ}$, with some small distortion due to the dressing laser. The distortion, however, is almost invisible, since the interference effect through LICS is negligible at far-off resonance. In Figs. 6(b) and 6(c), we see that the 3D PAD's are significantly modified. Especially in Fig. 6(c), a maximum distortion is observed in the PAD due to the strong destructive interference between the $\epsilon s$ and $\epsilon d$ partial waves.

\section{B. K $4 p_{3 / 2}-6 p_{3 / 2}$ system}

We now turn to the case of the $4 p_{3 / 2}-6 p_{3 / 2}$ system. This system is somehow different from the $4 p_{1 / 2}-6 p_{1 / 2}$ system, because the initial state $4 p_{3 / 2}$ is not spherically symmetric: Only $m_{j}= \pm 1 / 2$ out of all possible $m_{j}= \pm 1 / 2, \pm 3 / 2$ magnetic sublevels are equally occupied by the auxiliary laser, and for this reason a different behavior is expected.

In Fig. 7(a) we plot the variation of the total ionization yield as a function of two-photon detuning at four different
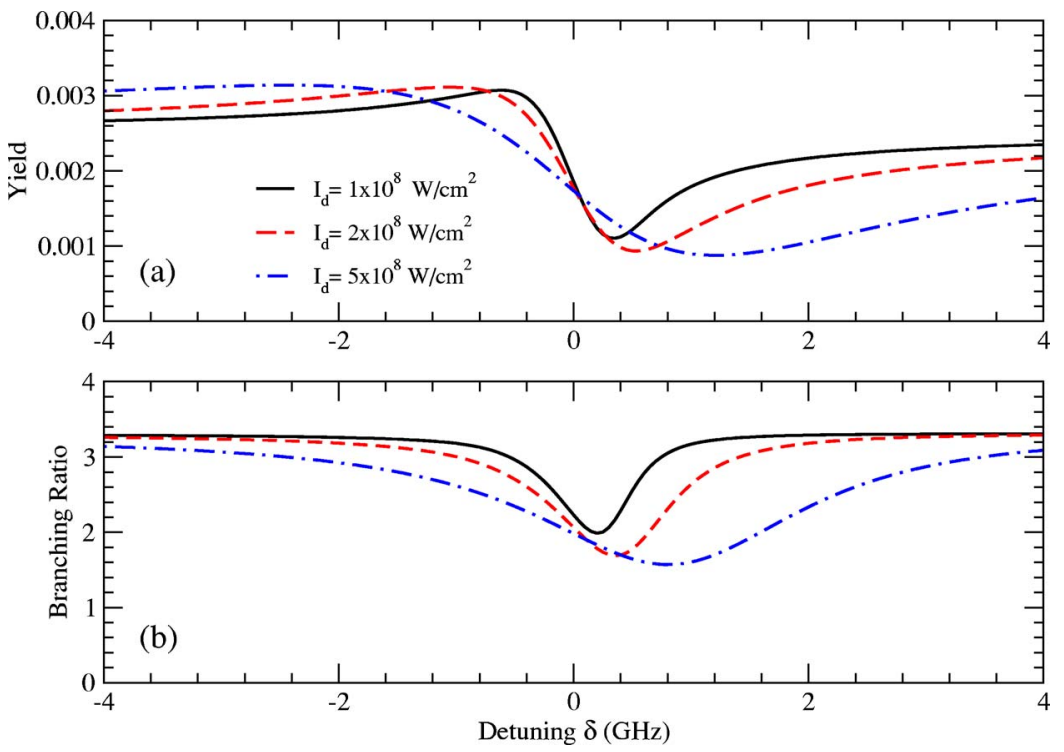

FIG. 8. (Color online) Same as in Fig. 4 but for the $\mathrm{K} 4 p_{3 / 2}-6 p_{3 / 2}$ system. 
(a)

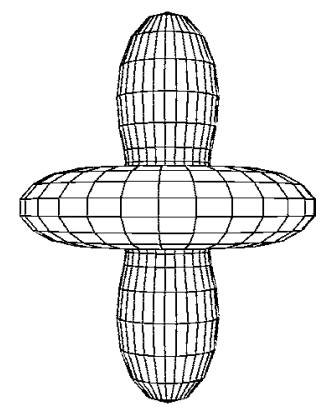

(b)

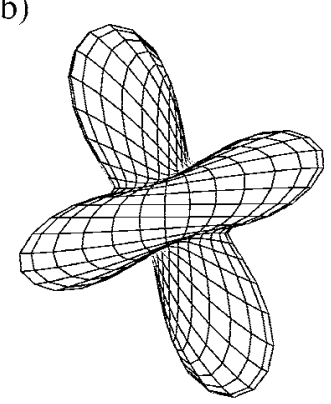

(c)

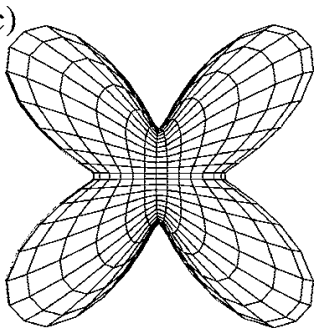

FIG. 9. Same as in Fig. 5 but for the $\mathrm{K} 4 p_{3 / 2}-6 p_{3 / 2}$ system. The viewpoint is from the positive $y$ axis.

values of the polarization angle, $\theta_{p}=0^{\circ}, 30^{\circ}, 60^{\circ}$, and $90^{\circ}$. The LICS structure in Fig. 7(a) is not quite similar to that plotted in Fig. 3(a), because, although $q_{13}^{\epsilon s}$ and $q_{13}^{\epsilon d}$ are the same for both systems, $q_{13}$ itself is different. More interestingly, the variation of the branching ratios shown in Fig. 7(b) is substantially larger than that shown in Fig. 3(b) as $\theta_{p}$ increases. For $\theta_{p}=90^{\circ}$ the ionization yield into the $\epsilon d$ continuum at the detuning close to $\delta=-0.9 \mathrm{GHz}$ is almost 40 times enhanced compared to that into the $\epsilon s$ continuum. This suggests that an appropriate choice of the probe polarization angle and the two-photon detuning leads to the control of ionization into different channels. Recent experiments [17] performed for ionization from an excited state of Xe with linearly and circularly polarized lasers have demonstrated that the ionization products into different continua can be separated by varying the polarization of lasers. The variation of the total ionization yields and the branching ratios as a function of detuning $\delta$ at three different dressing laser intensities, $I_{d}=100,200$, and $500 \mathrm{MW} / \mathrm{cm}^{2}$, is presented in Figs. 8(a) and 8(b) with the rest of the parameters being the same as those in Fig. 4.

In Figs. 9(a)-9(c) we plot the 3D PAD for one-photon ionization from the $4 p_{3 / 2}$ state by the probe laser at $\theta_{p}=0^{\circ}$, $45^{\circ}$, and $90^{\circ}$, without the dressing laser-i.e., $I_{d}=0$. Com- (a)

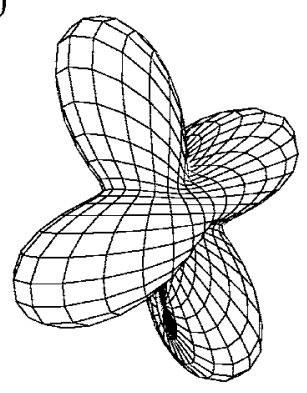

(b)

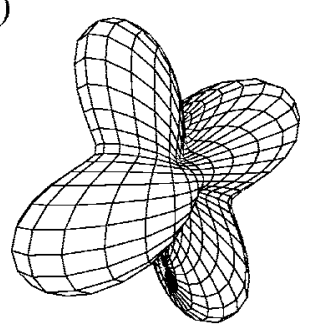

(c)

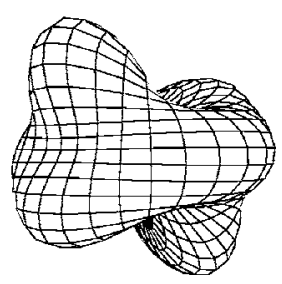

FIG. 10. Three-dimensional photoelectron angular distribution for the $\mathrm{K} 4 p_{3 / 2}-6 p_{3 / 2}$ system at three different two-photon detunings $\delta=-4,-0.89$, and $0.42 \mathrm{GHz}$. Pulse durations and peak intensities are $\tau_{p}=1 \mathrm{~ns}$ and $I_{p}=1 \mathrm{MW} / \mathrm{cm}^{2}$ for the probe laser and $\tau_{d}=10 \mathrm{~ns}$ and $I_{p}=100 \mathrm{MW} / \mathrm{cm}^{2}$ for the dressing laser. The polarization angle is $\theta_{p}=60^{\circ}$.

pared to the $4 p_{1 / 2}-6 p_{1 / 2}$ system [see Figs. 5(a)-5(c)], the PAD's drastically change the shape with detuning when the probe polarization angle is varied.

Now we return to the case for LICS by turning on the dressing laser and present the 3D PAD's for the $4 p_{3 / 2}-6 p_{3 / 2}$ system in Figs. $10(\mathrm{a})-10(\mathrm{c})$, at $\theta_{p}=60^{\circ}$, for three representative detunings corresponding to the far-off resonance $(\delta=-4 \mathrm{GHz})$, maximum $(\delta=-0.89 \mathrm{GHz})$, and minimum $(\delta=0.42 \mathrm{GHz})$ of the branching ratio [see Fig. 7(b)]. The modification of the 3D PAD's, presented in Figs. 9 and 10, is more than we expect: The variation of the sidelobes of $3 \mathrm{D}$ PAD's at different polarization angles, which are absent for the $4 p_{1 / 2}-6 p_{1 / 2}$ system, is striking. The sidelobes are due to the ionization into the $\epsilon d_{5 / 2}$ continuum (which is inaccessible through one-photon ionization from the $4 p_{1 / 2}$ state) and are present even if $\theta_{p}=0^{\circ}$.

As we have already noticed, not only the photoelectron angular distribution but also the angle-integrated ionization yield is affected by the relative polarization angle between the probe and dressing lasers. This effect is called linear dichroism (LD) and is very attractive from the experimental point of view, since it is much easier to measure the total ionization yield than the PAD. Linear dichroism can be experimentally used to determine the ratio of the dipole matrix 

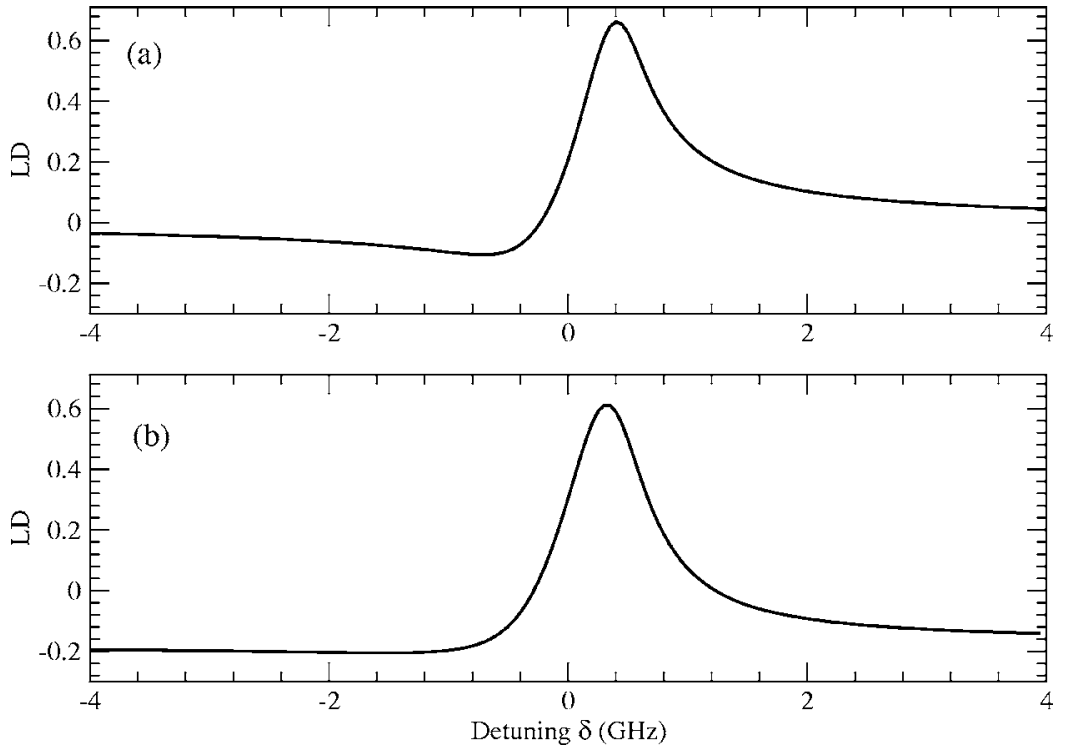

elements into the different continua [18] or the relative phase shift between the partial waves of the continua. The normalized linear dichroism is defined as [19]

$$
\mathrm{LD}=\frac{R\left(\theta_{p}=90^{\circ}\right)-R\left(\theta_{p}=0^{\circ}\right)}{R\left(\theta_{p}=90^{\circ}\right)+R\left(\theta_{p}=0^{\circ}\right)},
$$

where $R\left(\theta_{p}=0^{\circ}\right)$ and $R\left(\theta_{p}=90^{\circ}\right)$ represent the total ionization yield or, equivalently, the angle-integrated photoelectron signal when the polarization axis of the probe laser is parallel and perpendicular with respect to that of the dressing laser, respectively. In Figs. 11(a) and 11(b) we plot the linear dichroism as a function of two-photon detuning for the $\mathrm{K}$ $4 p_{1 / 2}-6 p_{1 / 2}$ and $4 p_{3 / 2}-6 p_{3 / 2}$ systems, respectively. The magnitude of the linear dichroism changes drastically for both systems around the LICS resonance, and it shows a large maximum for the two-photon detunings around the deep LICS minimum at $\theta_{p}=0^{\circ}$.

\section{SUMMARY}

In this paper we have theoretically investigated the effects of the relative polarization angle between the probe and dressing lasers on the total (angle-integrated) ionization yield, branching ratio, and PAD through LICS for the $\mathrm{K}$ $4 p_{1 / 2}-6 p_{1 / 2}$ and $4 p_{3 / 2}-6 p_{3 / 2}$ systems in a particular geometry with both probe and dressing lasers being linearly polarized. Amplitude equation and alternatively density matrix equation formalisms have been used to study the dynamics of the ionization process. We have shown that the ionization yield and the branching ratio are strongly dependent on the relative polarization angle between the lasers. Moreover, we have found that ionization into the different continua, branching ratios, and PAD's is significantly altered by the change of the polarization angle. Our findings suggest that the relative polarization angle can be another doorknob to control the ionization dynamics through LICS. We have also calculated linear dichroism for the angle-integrated ionization yield, which turned out to be quite large at the two-photon detunings close to the LICS minimum. $\sigma:$
FIG. 11. Linear dichroism for the (a) $\mathrm{K}$ $4 p_{1 / 2}-6 p_{1 / 2}$ system and (b) $\mathrm{K} 4 p_{3 / 2}-6 p_{3 / 2}$ system as a function of two-photon detuning $\delta$. Pulse durations and peak intensities are $\tau_{p}=1 \mathrm{~ns}$ and $I_{p}$ $=1 \mathrm{MW} / \mathrm{cm}^{2}$ for the probe laser and $\tau_{d}=10 \mathrm{~ns}$ and $I_{p}=100 \mathrm{MW} / \mathrm{cm}^{2}$ for the dressing laser.

\section{ACKNOWLEDGMENTS}

G.B. acknowledges financial support from Japan Society for the Promotion of Science (JSPS). The work by T.N. was supported by a Grant-in-Aid for scientific research from the Ministry of Education and Science of Japan.

\section{APPENDIX A: TIME-DEPENDENT DENSITY MATRIX EQUATIONS}

Based on the density matrix approach [20], we study the temporal evolution of the $\mathrm{K}$ atom in the laser field given by Eq. (1). Briefly we solve the following set of time-dependent differential equations for the slowly varying density matrix

$$
\begin{aligned}
& \dot{\sigma}_{i i}=-\widetilde{\Gamma}_{i} \sigma_{i i}-2 \operatorname{Im}\left[\sum_{j=3}^{4} \Omega_{j i}\left(1+\frac{i}{q_{j i}}\right) \sigma_{i j}\right] \\
& \dot{\sigma}_{j j}=-\widetilde{\Gamma}_{j} \sigma_{j j}+2 \operatorname{Im}\left[\sum_{i=1}^{2} \Omega_{j i}\left(1-\frac{i}{q_{j i}}\right) \sigma_{i j}\right] \\
& \dot{\sigma}_{i j}=\left[i \delta_{i j}-\frac{1}{2}\left(\widetilde{\Gamma}_{i}+\widetilde{\Gamma}_{j}\right)\right] \sigma_{i j}+i \sum_{i^{\prime}=1}^{2} \Omega_{i^{\prime} j}\left(1-\frac{i}{q_{i^{\prime} j}}\right) \sigma_{i i^{\prime}} \\
& -i \sum_{j^{\prime}=3}^{4} \Omega_{i j^{\prime}}\left(1-\frac{i}{q_{i j^{\prime}}}\right) \sigma_{j^{\prime} j} \text {, } \\
& \dot{\sigma}_{i i^{\prime}}=-\frac{1}{2}\left(\widetilde{\Gamma}_{i}+\widetilde{\Gamma}_{i^{\prime}}\right) \sigma_{i i^{\prime}}+i \sum_{j=3}^{4} \Omega_{j i^{\prime}}\left(1+\frac{i}{q_{j i^{\prime}}}\right) \sigma_{i j} \\
& -i \sum_{j=3}^{4} \Omega_{i^{\prime} j}\left(1-\frac{i}{q_{i^{\prime} j}}\right) \sigma_{j i^{\prime}},
\end{aligned}
$$




$$
\begin{aligned}
\dot{\sigma}_{i j^{\prime}}= & -\frac{1}{2}\left(\widetilde{\Gamma}_{j}+\widetilde{\Gamma}_{j^{\prime}}\right) \sigma_{j j^{\prime}}+i \sum_{i=1}^{2} \Omega_{i j^{\prime}}\left(1+\frac{i}{q_{i j^{\prime}}}\right) \sigma_{j i} \\
& -i \sum_{i=1}^{2} \Omega_{j i}\left(1-\frac{i}{q_{j i}}\right) \sigma_{i j^{\prime}},
\end{aligned}
$$

where the indices take the values $i, i^{\prime}=1,2$ and $j, j^{\prime}=3,4$, with $i \neq i^{\prime}$ and $j \neq j^{\prime}$. All the density matrix elements for the continuum have been adiabatically eliminated from Eqs. (A1)-(A5). Note that we have used the rotating-wave approximation and the slowly varying density matrix elements to derive the above equations: $\sigma_{i i}=\rho_{i i}(i=1,4), \quad \sigma_{i j}$ $=\rho_{i j} e^{-i \delta_{\text {static }, i j} t}(i=1,2$ and $j=3,4), \sigma_{i c}=\rho_{i c} e^{-i \omega_{\alpha} t}$, and $(\alpha=p$ or $d$ and $i=1,4)$, where $\rho_{i j}(t)=u_{i}(t) u_{j}^{*}(t)$ are the density matrix elements. $\delta_{i j}$ is the two-photon detuning defined by $\delta_{i j}$ $=\delta_{\text {static }, i j}+\delta_{\text {stark }, i j}$, where the static detuning is defined by $\delta_{\text {static }, i j}=\left(E_{i}+\hbar \omega_{p}\right)-\left(E_{j}+\hbar \omega_{d}\right)$, and $\delta_{\text {stark }, i j}$ is the total dynamic ac Stark shift defined by $\delta_{\text {stark }, i j}=\left(S_{i}^{(p)}+S_{i}^{(d)}\right)-\left(S_{j}^{(p)}\right.$ $\left.+S_{j}^{(d)}\right)$. Now the above set of density matrix equations is solved with the following initial conditions: $\sigma_{i i}(t=-\infty)$ $=1 / 2$ and $\sigma_{j j}(t=-\infty)=\sigma_{i j}(t=-\infty)=0$, for $i=1,2$ and $j=3,4$. The total (angle-integrated) ionization yield is derived as

$$
\begin{aligned}
R(t)= & \int_{-\infty}^{t} d t^{\prime}\left\{\sum_{i=1}^{2} \Gamma_{i}^{(p)} \sigma_{i i}+\sum_{j=3}^{4}\left(\Gamma_{j}^{(d)}+\Gamma_{j}^{(p)}\right) \sigma_{j j}\right. \\
& \left.+4 \sum_{i=1}^{2} \sum_{j=3}^{4} \operatorname{Im}\left[\Omega_{j i}\left(1+\frac{i}{q_{j i}}\right)\right] \operatorname{Re}\left(\sigma_{i j}\right)\right\} .
\end{aligned}
$$

The probability that a photoelectron is ejected into a solid angle $\Omega_{\mathbf{k}}$ is given by the following formula:

$$
\begin{aligned}
& \frac{d R(\Theta, \Phi)}{d t d \Omega_{\mathbf{k}}} \\
& =0.589 \pi \sum_{m_{s^{\prime}}= \pm 1 / 2}\left\{\sum_{i=1}^{2} \Gamma_{i}^{\left(p, m_{s^{\prime}}\right)}(\Theta, \Phi) \sigma_{i i}\right. \\
& +\sum_{j=3}^{4}\left[\Gamma_{j}^{\left(d, m_{s^{\prime}}\right)}(\Theta, \Phi)+\Gamma_{j}^{\left(p, m_{s^{\prime}}\right)}(\Theta, \Phi)\right] \sigma_{j j} \\
& +2 \operatorname{Re}\left[\sum_{i=1}^{2} \sum_{j=3}^{4} \sqrt{\Gamma_{i}^{\left(p, m_{s^{\prime}}\right)}(\Theta, \Phi)} \sqrt{\left(\Gamma_{j}^{\left(p, m_{s^{\prime}}\right)}(\Theta, \Phi)\right)^{*}} \sigma_{i j}\right] \\
& +2 \operatorname{Re}\left[\sqrt{\Gamma_{1}^{\left(p, m_{s^{\prime}}\right)}(\Theta, \Phi)} \sqrt{\left(\Gamma_{2}^{\left(p, m_{s^{\prime}}\right)}(\Theta, \Phi)\right)^{*}} \sigma_{12}\right] \\
& +2 \operatorname{Re}\left[\sqrt{\Gamma_{3}^{\left(d, m_{s^{\prime}}\right)}(\Theta, \Phi)} \sqrt{\left(\Gamma_{4}^{\left(d, m_{s^{\prime}}\right)}(\Theta, \Phi)\right)^{*}} \sigma_{34}\right] \\
& \left.+2 \operatorname{Re}\left[\sqrt{\Gamma_{3}^{\left(p, m_{s^{\prime}}\right)}(\Theta, \Phi)} \sqrt{\left(\Gamma_{4}^{\left(p, m_{s^{\prime}}\right)}(\Theta, \Phi)\right)^{*}} \sigma_{34}\right]\right\}, \quad(\mathrm{A} 7)
\end{aligned}
$$

which can be shown to be equivalent to Eq. (30). The numerical results for both $\mathrm{K} 4 p_{1 / 2}-6 p_{1 / 2}$ and $4 p_{3 / 2}-6 p_{3 / 2}$ systems obtained in the density matrix and amplitude equations formalisms are, of course, identical.

\section{APPENDIX B: IONIZATION WIDTHS}

The partial ionization width from state $|j\rangle$ to the continuum $|c\rangle$ produced by laser $\alpha$ is defined as

$$
\Gamma_{j c}^{\left(\alpha, m_{s^{\prime}}\right)}(\Theta, \Phi)=2 \pi\left|D_{j c}^{\left(\alpha, m_{s^{\prime}}\right)}(\Theta, \Phi)\right|^{2},
$$

where $D_{j c}^{\left(\alpha, m_{s^{\prime}}\right)}=-\mathrm{E}_{\alpha}(t) \Sigma_{q= \pm 1,0}\left\langle c\left|r_{q} e_{q}^{(\alpha)}\right| j\right\rangle \equiv \mathrm{E}_{\alpha}(t) \mu_{j c}^{\left(\alpha, m_{s^{\prime}}\right)}$ represents the one-photon dipole matrix element between states $|j\rangle$ and $|c\rangle$, expressed in the length gauge and calculated at energy $E_{c}=E_{j}+\omega_{\alpha}$. The total ionization width integrated over the solid angle $\Omega_{\mathbf{k}}$, defined by the polar angles $(\Theta, \Phi)$, of the ejected photoelectron is given by

$$
\Gamma_{j}^{(\alpha)}=\sum_{m_{s^{\prime}}= \pm 1 / 2} \sum_{c} \Gamma_{j c}^{\left(\alpha, m_{s^{\prime}}\right)} .
$$

The summation over $c$ implies that the summation is taken over all allowed continuum states.

\section{APPENDIX C: ac STARK SHIFTS}

The dynamic ac Stark shift of the energy of state $|j\rangle$ due to both bound and free states $|k\rangle$, caused by laser $\alpha$, is

$$
S_{j}^{(\alpha)}=\sum_{m_{s^{\prime}}= \pm 1 / 2} \sum_{k}\left[\frac{\left|D_{j k}^{\left(\alpha, m_{s^{\prime}}\right)}\right|^{2}}{E_{j}+\omega_{\alpha}-E_{k}+i \varepsilon}+\frac{\left|D_{j k}^{\left(\alpha, m_{s^{\prime}}\right)^{2}}\right|^{2}}{E_{j}-\omega_{\alpha}-E_{k}+i \varepsilon}\right] .
$$

The sum here contains both summation over the bound and integration over the continuum states, and $\varepsilon$ is an infinitely small number.

\section{APPENDIX D: TWO-PHOTON RABI FREQUENCY}

The total two-photon Rabi frequency $\Omega_{i j}$ between states $|i\rangle$ and $|j\rangle$ is given by

$$
\Omega_{i j}\left(1-\frac{i}{q_{i j}}\right)=\sum_{c} \Omega_{i j}^{c}\left(1-\frac{i}{q_{i j}^{c}}\right),
$$

where the partial two-photon Rabi frequency between states $|i\rangle$ and $|j\rangle$ coupled through the continuum $|c\rangle$ with the energy $E_{1}+\omega_{p} \simeq E_{3}+\omega_{d}$ is defined as

$$
\Omega_{i j}^{c}\left(1-\frac{i}{q_{i j}^{c}}\right)=\sum_{m_{s^{\prime}}= \pm 1 / 2} \int d E_{c} \frac{D_{i c}^{\left(p, m_{s^{\prime}}\right)} D_{c j}^{\left(d, m_{s^{\prime}}\right)}}{E_{1}+\omega_{p}-E_{c}+i \varepsilon} .
$$

The imaginary part of the partial Rabi frequency is connected to the partial asymmetry parameter $q_{i j}^{c}$ and is given by

$$
\frac{\Omega_{i j}^{c}}{q_{i j}^{c}}=\left.\sum_{m_{s^{\prime}}= \pm 1 / 2} \pi D_{i c}^{\left(p, m_{s^{\prime}}\right)} D_{c j}^{\left(d, m_{s^{\prime}}\right)}\right|_{E_{c}=E_{1}+\omega_{p}} .
$$


[1] Y. L. Shao, D. Charalambidis, C. Fotakis, Jian Zhang, and P. Lambropoulos, Phys. Rev. Lett. 67, 3669 (1991).

[2] S. Cavalieri, F. S. Pavone, and M. Matera, Phys. Rev. Lett. 67, 3673 (1991).

[3] P. L. Knight, M. A. Lauder, and B. J. Dalton, Phys. Rep. 190, 1 (1991)

[4] O. Faucher, D. Charalambidis, C. Fotakis, J. Zhang, and P. Lambropoulos, Phys. Rev. Lett. 70, 3004 (1993); O. Faucher, Y. L. Shao, and D. Charalambidis, J. Phys. B 26, L309 (1993); O. Faucher, Y. L. Shao, D. Charalambidis, and C. Fotakis, Phys. Rev. A 50, 641 (1994).

[5] Takashi Nakajima and L. A. A. Nikolopoulos, Phys. Rev. A 68, 013413 (2003).

[6] K. Böhmer, T. Halfmann, L. P. Yatsenko, D. Charalambidis, A. Horsmans, and K. Bergmann, Phys. Rev. A 66, 013406 (2002).

[7] Z. Chen, M. Shapiro, and P. Brumer, Chem. Phys. Lett. 228, 289 (1994).

[8] P. Lambropoulos and M. R. Teague, J. Phys. B 9, 587 (1976); Zheng-Min Wang and D. S. Elliott, Phys. Rev. A 62, 053404 (2000); N. M. Kabachnik and K. Ueda, J. Phys. B 28, 5013 (1995).
[9] J. A. Duncanson, Jr., M. P. Strand, A. Lindgrd, and R. S. Berry, Phys. Rev. Lett. 37, 987 (1976).

[10] Takashi Nakajima, Phys. Rev. A 61, 041403(R) (2000).

[11] Takashi Nakajima and Gabriela Buica, Phys. Rev. A 71, 013413 (2005).

[12] I. I. Sobelman, Atomic Spectra and Radiative Transitions (Springer, Berlin, 1992).

[13] P. Lambropoulos, Phys. Rev. Lett. 30, 413 (1973).

[14] L.-W. He, C. E. Burkhardt, M. Ciocca, J. J. Leventhal, H.-L. Zhou, and S. T. Manson, Phys. Rev. A 51, 2085 (1995).

[15] S. Basile, F. Trombetta, and G. Ferrante, Phys. Rev. Lett. 61, 2435 (1988).

[16] M. Fifirig and V. Florescu, Eur. Phys. J. D 2, 143 (1998).

[17] S. Aloïse, P. O’Keeffe, D. Cubaynes, M. Meyer, and A. N. Grum-Grzhimailo, Phys. Rev. Lett. 94, 223002 (2005).

[18] A. von dem Borne, Th. Dohrmann, A. Verweyen, B. Sonntag, K. Godehusen, P. Zimmermann, and N. M. Kabachnik, J. Phys. B 31, L41 (1998).

[19] N. A. Cherepkov, V. V. Kuznetsov, and V. A. Verbitskii, J. Phys. B 28, 1221 (1995).

[20] S. N. Dixit and P. Lambropoulos, Phys. Rev. A 27, 861 (1983). 